PROCEEDINGS OF THE

AMERICAN MATHEMATICAL SOCIETY

Volume 127, Number 8, Pages 2289-2295

S 0002-9939(99)04858-3

Article electronically published on April 9, 1999

\title{
A BOUND FOR THE THEOREM OF DE FRANCHIS
}

\author{
MASAHARU TANABE \\ (Communicated by Albert Baernstein II)
}

\begin{abstract}
We give a bound on the number of nonconstant holomorphic maps between compact Riemann surfaces of genera $>1$.
\end{abstract}

\section{INTRODUCTION}

Holomorphic maps of Riemann surfaces have a lot of rigid properties. It is known that the action of a holomorphic map on the fundamental groups, or on the homology groups strongly restricts the behaviour of the holomorphic map. Using these rigid properties, we may show finiteness of the number of holomorphic maps in the case of compact Riemann surfaces.

Let $\widetilde{X}$ be a compact Riemann surface of genus $\tilde{g}(>1)$. Related to finiteness of the number of holomorphic maps, de Franchis $[\mathrm{F}]$ stated the following:

Theorem of de Franchis. (1) For a fixed compact Riemann surface $X$ of genus $>1$, the number of nonconstant holomorphic maps $\widetilde{X} \rightarrow X$ is finite.

(2) There are only finitely many compact Riemann surfaces $X_{i}$ of genus $>1$ which admit a nonconstant holomorphic map from $\widetilde{X}$.

For algebraic proofs of this theorem, see e.g. Kani [K], Martens [Mr2], [Mr3], and Howard and Sommese [H-S]. Imayoshi [I1] [I2] gave the analytic proofs for Riemann surfaces of finite types.

It is an interesting problem to find an upper bound of the number of nonconstant holomorphic maps of $\widetilde{X}$ to $X$. When these genera are the same, any nonconstant holomorphic map must be conformal. Thus the number of such holomorphic maps is just the number of holomorphic automorphisms of $\widetilde{X}$, and it is not greater than 84 $(\tilde{g}-1)$ by Hurwitz's theorem [H]. Furthermore, Machbeath [Mc] showed that the bound is sharp for an infinite number of integers $\tilde{g}$.

Let $g(>1)$ denote the genus of a target surface of a holomorphic map from $\tilde{X}$, and we consider the case that $\tilde{g}>g$. Let $h_{i}: \tilde{X} \rightarrow X_{i}$ be a nonconstant holomorphic map $(i=1,2)$. We say that $h_{1}$ and $h_{2}$ are isomorphic if and only if there is a conformal map $f: X_{1} \rightarrow X_{2}$ such that $f \circ h_{1}=h_{2}$. Let $\mathcal{I}_{g}(\widetilde{X})$ be the set of all isomorphic classes of nonconstant holomorphic maps into compact Riemann surfaces of genus $g>1$, and let $\mathcal{I}(\tilde{X})=\bigcup_{\tilde{g}>g>1} \mathcal{I}_{g}(\tilde{X})$. By the theorem of de Franchis, we see that $\sharp \mathcal{I}(\widetilde{X})$ is finite. Howard and Sommese [H-S] first showed that

Received by the editors August 19, 1997 and, in revised form, October 27, 1997.

1991 Mathematics Subject Classification. Primary 30F30.

(C)1999 American Mathematical Society 
there is a bound on $\sharp \mathcal{I}(\widetilde{X})$ depending only on $\tilde{g}$. Kani $[\mathrm{K}]$ showed that

$$
\sharp \mathcal{I}_{g}(\widetilde{X})<2^{2 \tilde{g}^{2}-1}\left(2^{2 \tilde{g}^{2}-1}-1\right),
$$

and

$$
\sharp \mathcal{I}(\widetilde{X})<(\tilde{g}-1) 2^{2 \tilde{g}^{2}-2}\left(2^{2 \tilde{g}^{2}-1}-1\right) .
$$

To the best knowledge of the author, it is the smallest bound depending only on $\tilde{g}$.

In this paper, we will estimate the number of nonconstant holomorphic maps of $\tilde{X}$ into a fixed Riemann surface $X$ of genus $g$. In this case, Martens [Mr2] gave the first proof that the number of all nonconstant holomorphic maps into a fixed Riemann surface has a bound depending only on $\tilde{g}$ (explicitly computable and it seems that the bound was given by $(c \tilde{g})^{2 \tilde{g}^{2}}$ for some constant $c$ independent of $\tilde{g})$. Using Kani's estimate, we see immediately that the number of all nonconstant holomorphic maps into a fixed Riemann surface $X$ of genus $g>1$ is less than

$$
2^{2 \tilde{g}^{2}-1}\left(2^{2 \tilde{g}^{2}-1}-1\right) \times 84(g-1)
$$

where $84(g-1)$ is the upper bound of $\sharp A u t(X)$. It was the smallest bound depending only on $\tilde{g}$ and $g$ when the author started to consider this problem, although Kani's estimate is counting maps into other Riemann surfaces of the same genus $g$.

Here, we will show

Theorem. Let $\tilde{X}, X$ be compact Riemann surfaces of genera $\tilde{g}, g(>1)$. Let $\operatorname{Hol}(\widetilde{X}, X)$ be the set of all nonconstant holomorphic maps of $\widetilde{X}$ into $X$. Then, we have

$$
\sharp \operatorname{Hol}(\tilde{X}, X) \leq(4(\tilde{g}-1) /(g-1)+1)^{2 \tilde{g}} \times 2(\tilde{g}-1) \times(2 g-1) .
$$

If one wants to have a bound without using $g$, one may write

$$
\sharp \operatorname{Hol}(\widetilde{X}, X) \leq(4 \tilde{g}-3)^{2 \tilde{g}} \times 6(\tilde{g}-1)
$$

since the left-hand side of the inequality of the Theorem is monotone decreasing for $g>1$.

By the Theorem, we may say that $\sharp \operatorname{Hol}(\tilde{X}, X) \leq(c \tilde{g})^{2 \tilde{g}}$ for some constant $c$, while $\sharp \mathcal{I}(\widetilde{X}) \leq c^{\prime \tilde{g}^{2}}$ for some constant $c^{\prime}$ by Kani's estimate.

\section{Preliminaries}

Let $\widetilde{X}, X$ be compact Riemann surfaces of genera $\tilde{g}, g(>1)$. We denote by $H_{1}(X)$ the first homology group with $\mathbb{Z}$-coefficients of $X$. Any basis for $H_{1}(X)$ (say $\left\{\chi_{1}, \ldots, \chi_{2 g}\right\}$ ), with intersection matrix (that is a matrix whose $(k, j)$ )-entry is given by the intersection number $\chi_{k} \cdot \chi_{j}$ ),

$$
J=\left(\begin{array}{cc}
0 & E \\
-E & 0
\end{array}\right)
$$

will be called a canonical homology basis, where $E$ is the $g \times g$ identity matrix.

We denote by $H$ the space of harmonic differentials on $X$. For a canonical homology basis $\left\{\chi_{1}, \ldots, \chi_{2 g}\right\}$, there is a unique dual basis $\left\{\alpha_{1}, \ldots, \alpha_{2 g}\right\}\left(\int_{\chi_{j}} \alpha_{k}=\right.$ $\left.\delta_{j k}(j, k=1, \ldots, 2 g)\right)$ of $H$, where each $\alpha_{k}$ is real. Similarly for $\tilde{X}$.

Let $h: \widetilde{X} \rightarrow X$ be a nonconstant holomorphic map. Then $h$ induces a homomorphism $h_{*}: H_{1}(\tilde{X}) \rightarrow H_{1}(X)$. Let $M=\left(m_{k j}\right) \in M(2 g, 2 \tilde{g} ; \mathbb{Z})$, where $h_{*}\left(\tilde{\chi}_{j}\right)=\sum_{k=1}^{2 g} m_{k j} \chi_{k}$. (We denote by $M(m, n ; K)$ the set of $m \times n$ matrices 
with $K$-coefficients.) We will call $M$ the matrix representation of $h$ with respect to $\left\{\tilde{\chi}_{1}, \ldots, \tilde{\chi}_{2 \tilde{g}}\right\}$ and $\left\{\chi_{1}, \ldots, \chi_{2 g}\right\}$. We have another interpretation of $M$. Considering an equality $\int_{\tilde{\chi}_{j}} \alpha_{k} \circ h=\int_{h_{*}\left(\tilde{\chi}_{j}\right)} \alpha_{k}$, we may write the pull-back of $\alpha_{k}$ as

$$
\alpha_{k} \circ h=\sum_{j=1}^{2 \tilde{g}} m_{k j} \tilde{\alpha}_{j} .
$$

Thus, we may consider $M$ as the representation of $h$ on the space of harmonic differentials.

We denote by $*$ the conjugation operator, that is, for a 1 -form $w=f d x+g d y(z=$ $x+i y$ is a local coordinate)

$$
{ }^{*} w=-g d x+f d y
$$

We represent $*$ by a $2 g \times 2 g($ resp. $2 \tilde{g} \times 2 \tilde{g})$ real matrix $\mathcal{G}($ resp. $\widetilde{\mathcal{G}})$ with respect to the basis $\left\{\alpha_{1}, \ldots, \alpha_{2 g}\right\}\left(\left\{\tilde{\alpha}_{1}, \ldots, \tilde{\alpha}_{2 g}\right\}\right)$ of $H$ (resp. $\left.\widetilde{H}\right)$,

$$
\begin{gathered}
\mathcal{G}=\left(g_{j k}\right) \quad(j, k=1, \ldots, 2 g), \\
\text { (resp. } \left.\widetilde{\mathcal{G}}=\left(\tilde{g}_{j k}\right) \quad(j, k=1, \ldots, 2 \tilde{g})\right),
\end{gathered}
$$

that is, ${ }^{*} \alpha_{k}=\sum_{j=1}^{2 g} g_{j k} \alpha_{j}$ (resp. ${ }^{*} \tilde{\alpha}_{k}=\sum_{j=1}^{2 \tilde{g}} \tilde{g}_{j k} \tilde{\alpha}_{j}$ ). It is easy to see that $\mathcal{G}^{2}=-E$. Let a $2 g \times 2 g$ real matrix

$$
\Gamma=\left(\gamma_{j k}\right)=\left(\int_{X} \alpha_{j} \wedge^{*} \alpha_{k}\right) \quad(j, k=1, \ldots, 2 g) .
$$

It is well-known that $\Gamma$ is symmetric, positive definite, and $\Gamma=J \mathcal{G}$ (see e.g. [F-K, chapter 3]). Similarly for $\tilde{X}$.

For an arbitrary 1-form $w$ on $X$, we have

$$
{ }^{*} w \circ h={ }^{*}(w \circ h) .
$$

For $\left\{\tilde{\alpha}_{1}, \ldots, \tilde{\alpha}_{2 \tilde{g}}\right\}$ and $\left\{\alpha_{1}, \ldots, \alpha_{2 g}\right\}$, considering the matrix representation of the above equality, we obtain

$$
M^{t} \widetilde{\mathcal{G}}={ }^{t} \mathcal{G} M
$$

$\left({ }^{t} \mathcal{G}\right.$ denotes the transposition of $\mathcal{G}$.) Indeed,

$$
{ }^{*}\left(\alpha_{j} \circ h\right)=*\left(\sum_{k}^{2 \tilde{g}} m_{j k} \tilde{\alpha}_{k}\right)=\sum_{k} m_{j k}{ }^{*} \tilde{\alpha}_{k}=\sum_{k} m_{j k}\left(\sum_{l=1}^{2 \tilde{g}} \tilde{g}_{l k} \tilde{\alpha}_{l}\right)=\sum_{l} \sum_{k} m_{j k} \tilde{g}_{l k} \tilde{\alpha}_{l},
$$

and

$$
{ }^{*} \alpha_{j} \circ h=\left(\sum_{k} g_{k j} \alpha_{k}\right) \circ h=\sum_{k} g_{k j}\left(\sum_{l} m_{k l} \tilde{\alpha}_{l}\right)=\sum_{l} \sum_{k} g_{k j} m_{k l} \tilde{\alpha}_{l} .
$$

Since the equality ${ }^{*}\left(\alpha_{j} \circ h\right)={ }^{*} \alpha_{j} \circ h$ holds, we have (1).

It is easy to see that the set of all $M \in M(2 g, 2 \tilde{g} ; \mathbb{Z})$ satisfying (1) is a $\mathbb{Z}$-module. We denote it by $\mathcal{M}(\widetilde{\mathcal{G}}, \mathcal{G})$.

Remark. For the case that $M$ is $2 g \times 2 g$ sized, that is, $M^{t} \mathcal{G}={ }^{t} \mathcal{G} M$, Weyl [W] investigated the relation algebraically to give conditions that $M$ satisfies for a given $\mathcal{G}$. 
Lemma 1. Let $D \in \mathcal{M}(\widetilde{\mathcal{G}}, \mathcal{G})$. Then, we have

$$
D \widetilde{\Gamma}^{t} D \Gamma^{-1}=D \widetilde{J}^{t} D J^{-1}
$$

In particular, when $M$ is the matrix representation of a nonconstant holomorphic map $h: \widetilde{X} \rightarrow X$ of degree $d$,

$$
M \widetilde{\Gamma}^{t} M \Gamma^{-1}=M \widetilde{J}^{t} M J^{-1}=d E .
$$

Proof. Indeed,

$$
D \widetilde{\Gamma}^{t} D \Gamma^{-1}=D \widetilde{J} \widetilde{\mathcal{G}}^{t} D \Gamma^{-1}=D \widetilde{J}^{t} D \mathcal{G} \Gamma^{-1}=D \widetilde{J}^{t} D J^{-1}
$$

since $\Gamma=J \mathcal{G}={ }^{t} \mathcal{G}^{t} J$. When $M$ is the matrix representation of a nonconstant holomorphic map of degree $d$, equality $M \widetilde{J}^{t} M J^{-1}=d E$ holds (see e.g. [Mr3], [T]). Thus, we have (3).

Remark. If $\tilde{X}$ and $X$ are conformally equivalent, a matrix $M \in M(2 g, 2 g ; \mathbb{Z})$ exists which satisfies formula (3) (Torelli's theorem, see e.g. [Mr1]).

Lemma 2. Let

$$
D^{\prime}=\widetilde{J}^{t} D J^{-1} D,
$$

where $D \in \mathcal{M}(\widetilde{\mathcal{G}}, \mathcal{G})$. Then we have

$$
D^{t} \widetilde{\mathcal{G}}={ }^{t} \widetilde{\mathcal{G}} D^{\prime},
$$

and

$$
D^{\prime} \widetilde{\Gamma}^{t} D^{\prime} \widetilde{\Gamma}^{-1}=D^{\prime} \widetilde{J}^{t} D^{\prime} \widetilde{J}^{-1}
$$

In particular, when $M^{\prime}=\widetilde{J}^{t} M J^{-1} M$ where $M$ is the matrix representation of a nonconstant holomorphic map $h: \widetilde{X} \rightarrow X$ of degree $d$, we have

$$
M^{\prime} \widetilde{\Gamma}^{t} M^{\prime} \widetilde{\Gamma}^{-1}=d M^{\prime} .
$$

Proof. Indeed,

$$
\begin{aligned}
D^{\prime}{ }^{t} \widetilde{\mathcal{G}} & =\widetilde{J}^{t} D J^{-1} D^{t} \widetilde{\mathcal{G}}=\widetilde{J}^{t} D J^{-1}{ }^{t} \mathcal{G} D \\
& =\widetilde{J}^{t} D \mathcal{G} J D=\widetilde{\mathcal{G}}{ }^{t} D J D={ }^{t} \widetilde{\mathcal{G}} \widetilde{J}^{t} D J^{-1} D={ }^{t} \widetilde{\mathcal{G}} D^{\prime} .
\end{aligned}
$$

By the same consideration as in the proof of relation (1), we have (5). When $M^{\prime}=$ $\widetilde{J}^{t} M J^{-1} M$ where $M$ is the matrix representation of a nonconstant holomorphic map of degree $d, M \widetilde{J}^{t} M J^{-1}=d E$ holds and we see (6) holds by easy calculation.

Definition. Define the constant $I(X)$ by

$$
I(X)=\inf _{\mathbf{n} \in \mathbb{Z}^{2 g} \backslash\{\mathbf{0}\}} \mathbf{n} \Gamma^{t} \mathbf{n} .
$$

It is easy to see that $I(X)$ does not depend on the choice of the canonical homology basis. We see that $I(X)=\min _{\mathbf{n} \in \mathbb{Z}^{2 g} \backslash\{\mathbf{0}\}} \mathbf{n} \Gamma^{t} \mathbf{n}$ since $\Gamma$ is positive definite. We define a norm $|\cdot|_{\Gamma}$ by

$$
|\mathbf{n}|_{\Gamma}^{2}=\mathbf{n} \Gamma^{t} \mathbf{n} \quad\left(\mathbf{n} \in \mathbb{Z}^{2 g}\right) .
$$




\section{Proof of the Theorem}

Before proving the Theorem, we give a proposition.

Proposition. Let $h_{i}: \widetilde{X} \rightarrow X$ be a nonconstant holomorphic map, and $M_{i}$ be the matrix representation $(i=1,2)$. Let $e=\sum_{j=1}^{2 g} e_{j} \alpha_{j}\left(\mathbf{e}=\left(e_{j}\right) \in \mathbb{Z}^{2 g} \backslash\{\mathbf{0}\}\right)$ be a harmonic differential with $\mathbf{e} \Gamma^{t} \mathbf{e}=I(X)$. Suppose that there is an integer $l>4(\tilde{g}-1) /(g-1)$ such that $\mathbf{e} M_{1} \equiv \mathbf{e} M_{2}($ mod. $l)$ holds. Then $e \circ h_{1}=e \circ h_{2}$.

Proof. Let $D=M_{2}-M_{1}$. Then,

$$
\mathbf{e} D \widetilde{J}^{t} D J^{-1} \equiv \mathbf{0} \quad(\bmod . l)
$$

by the assumption. We will first show that the equality (7) implies $\mathbf{e} D \widetilde{J}^{t} D J^{-1}=\mathbf{0}$. Let $\tilde{\mathbf{e}}=\mathbf{e} D$. If $\tilde{\mathbf{e}} \widetilde{J}^{t} D J^{-1} \neq \mathbf{0}$, then we have

$$
\begin{aligned}
l^{2} I(X) & \leq\left|\tilde{\mathbf{e}} \widetilde{J}^{t} D J^{-1}\right|_{\Gamma}^{2} \\
& \leq\left\{\left|\tilde{\mathbf{e}} \widetilde{J}^{t} M_{2} J^{-1}\right|_{\Gamma}+\left|\tilde{\mathbf{e}} \widetilde{J}^{t} M_{1} J^{-1}\right|_{\Gamma}\right\}^{2} .
\end{aligned}
$$

The second inequality is obtained by using the triangle inequality. By formula (3) in Lemma 1, we have

$$
\left|\tilde{\mathbf{e}} \widetilde{J}^{t} M_{i} J^{-1}\right|_{\Gamma}^{2}=d_{i}^{-1}\left|\tilde{\mathbf{e}} M_{i}^{\prime}\right|_{\widetilde{\Gamma}}^{2} \quad(i=1,2) .
$$

Thus,

$$
l^{2} I(X) \leq\left(d_{2}^{-1 / 2}\left|\tilde{\mathbf{e}} M_{2}^{\prime}\right|_{\widetilde{\Gamma}}+d_{1}^{-1 / 2}\left|\tilde{\mathbf{e}} M_{1}^{\prime}\right|_{\widetilde{\Gamma}}\right)^{2}
$$

Here we have

$$
\left|\tilde{\mathbf{e}} M_{i}^{\prime}\right|_{\widetilde{\Gamma}} \leq d_{i}|\tilde{\mathbf{e}}|_{\widetilde{\Gamma}}
$$

To show this, we will take a normal orthogonal basis in $\mathbb{R}^{2 \tilde{g}}$ with respect to the inner product defined by $\widetilde{\Gamma}$. First, let $\mathbf{u}_{1}=\tilde{\mathbf{e}} /|\tilde{\mathbf{e}}|$. Next, we apply the Schmidt orthogonalization to obtain a normal orthogonal basis $\left\{\mathbf{u}_{1}, \ldots, \mathbf{u}_{2 \tilde{g}}\right\}$. Then, we can write

$$
\tilde{\mathbf{e}} M_{i}^{\prime}=\sum_{j=1}^{2 \tilde{g}} x_{i j} \mathbf{u}_{j}, \quad x_{i j} \in \mathbb{R}(j=1, \ldots, 2 \tilde{g}) .
$$

By formula (6) in Lemma 2 , we have $\tilde{\mathbf{e}} M_{i}^{\prime} \widetilde{\Gamma}{ }^{t} M_{i}^{\prime}{ }^{t} \tilde{\mathbf{e}}=d_{i} \tilde{\mathbf{e}} M_{i}^{\prime} \widetilde{\Gamma^{t}} \tilde{\mathbf{e}}$, or

or

$$
\sum_{j=1}^{2 \tilde{g}} x_{i j}^{2}=d_{i}|\tilde{\mathbf{e}}| x_{i 1}
$$

$$
\left(x_{i 1}-d_{i}|\tilde{\mathbf{e}}| / 2\right)^{2}+\sum_{j=2}^{2 \tilde{g}} x_{i j}^{2}=\left(d_{i}|\tilde{\mathbf{e}}| / 2\right)^{2} .
$$

Hence, $\left(x_{i 1}, \ldots, x_{i 2 \tilde{g}}\right)$ must be on a $2 \tilde{g}$-1-dimensional sphere whose radius is $d_{i}|\tilde{\mathbf{e}}| / 2$, centre is $\left(d_{i}|\tilde{\mathbf{e}}| / 2,0, \ldots, 0\right)$. Thus, $\left|\tilde{\mathbf{e}} M_{i}^{\prime}\right|_{\widetilde{\Gamma}}^{2}=\sum_{j=1}^{2 \tilde{g}} x_{i j}^{2} \leq\left(d_{i}|\tilde{\mathbf{e}}|_{\widetilde{\Gamma}}\right)^{2}$.

Next, we will give an upper bound of $\tilde{\mathbf{e}}$. By the triangle inequality, we have

$$
|\tilde{\mathbf{e}}|_{\widetilde{\Gamma}}=|\mathbf{e} D|_{\widetilde{\Gamma}} \leq\left|\mathbf{e} M_{1}\right|_{\widetilde{\Gamma}}+\left|\mathbf{e} M_{2}\right|_{\widetilde{\Gamma}}
$$

By formula (3),

$$
\left|\mathbf{e} M_{i}\right|_{\widetilde{\Gamma}}^{2}=d_{i}|\mathbf{e}|_{\Gamma}^{2}=d_{i} I(X) \quad(i=1,2)
$$


holds. Combining (10) and (11), we obtain

$$
|\tilde{\mathbf{e}}|_{\widetilde{\Gamma}}^{2} \leq 4 d I(X),
$$

where $d=\max .\left(d_{1}, d_{2}\right)$. By applying (9) and (12) to (8),

$$
l^{2} I(X) \leq 4^{2} d^{2} I(X) .
$$

Therefore, we must have $l \leq 4 d \leq 4(\tilde{g}-1) /(g-1)$ (the right-hand side is obtained by the Riemann-Hurwitz formula), but it contradicts the assumption. Consequently, we see that (7) implies $\mathbf{e} D \widetilde{J}^{t} D J^{-1}=\mathbf{0}$.

Using formula (2), we have

$$
\mathbf{e} D \widetilde{\Gamma}^{t} D^{t} \mathbf{e}=\mathbf{e} D \widetilde{J}^{t} D J^{-1} \Gamma^{t} \mathbf{e}=0 .
$$

This implies that $\mathbf{e} D=\mathbf{0}$ or $e \circ h_{1}=e \circ h_{2}$ since $\widetilde{\Gamma}$ is positive definite.

Now, we prove the Theorem.

Proof of the Theorem. First, recall that, for an arbitrary 1-form $w$ on $X$, we have

$$
{ }^{*} w \circ h={ }^{*}(w \circ h),
$$

where $h \in \operatorname{Hol}(\widetilde{X}, X)$. Then, letting

$$
\phi=e+i^{*} e,
$$

we have

$$
e \circ h_{i}=e \circ h_{j} \Leftrightarrow \phi \circ h_{i}=\phi \circ h_{j},
$$

where $h_{i}, h_{j} \in \operatorname{Hol}(\widetilde{X}, X)$. Thus,

$$
\sharp\left\{\phi \circ h_{i} \mid h_{i} \in \operatorname{Hol}(\tilde{X}, X)\right\} \leq(4(\tilde{g}-1) /(g-1)+1)^{2 \tilde{g}}
$$

by the Proposition.

Let $p_{0} \in X$ be one of the zeroes of $\phi$. Suppose that $\phi \circ h_{i}=\phi \circ h_{j}$ and $h_{i}^{-1}\left(p_{0}\right) \cap h_{j}^{-1}\left(p_{0}\right) \neq \emptyset$. Then, let $\tilde{p}_{0}$ be such a zero of $\tilde{\phi}=\phi \circ h_{i}$. There are at most $2(\tilde{g}-1)$ possible points that $\tilde{p}_{0}$ can be since the number of zeroes of a holomorphic differential is $2(\tilde{g}-1)$ with multiplicity. Taking sufficiently small neighbourhood $U_{\tilde{p}_{0}}$ (resp. $U_{p_{0}}$ ) of $\tilde{p}_{0}$ (resp. $p_{0}$ ), we may assume that there is no zero of $\tilde{\phi}$ (resp. $\phi$ ) on $U_{\tilde{p}_{0}}$ (resp. $\left.U_{p_{0}}\right)$ except $\tilde{p}_{0}\left(\right.$ resp. $\left.p_{0}\right)$, and $h_{k}\left(U_{\tilde{p}_{0}}\right) \subset U_{p_{0}}(k=i, j)$. We may take a local coordinate $\tilde{z}\left(\right.$ resp. $z$ ) on $U_{\tilde{p}_{0}}$ (resp. $U_{p_{0}}$ ) such that $\tilde{p}_{0}$ (resp. $p_{0}$ ) corresponds to 0 (resp. 0) and

$$
\tilde{\phi}=\tilde{z}^{m} d \tilde{z} \quad\left(\operatorname{resp} . \phi=z^{n} d z\right) .
$$

For an arbitrary $\tilde{p} \in U_{\tilde{p}_{0}} \backslash\left\{\tilde{p}_{0}\right\}$,

$$
\int_{0}^{\tilde{p}} \tilde{z}^{m} d \tilde{z}=\int_{0}^{h_{i}(\tilde{p})} z^{n} d z=\int_{0}^{h_{j}(\tilde{p})} z^{n} d z
$$

Thus, there are at most $n+1 \leq 2 g-1$ possible points on $U_{p_{0}}$ that (14) holds. By the identity theorem, $h_{i}(\tilde{p})=h_{j}(\tilde{p})$ for an arbitrary $\tilde{p} \in U_{\tilde{p}_{0}} \backslash\left\{\tilde{p}_{0}\right\}$ implies $h_{i}=h_{j}$.

Let us sum up the argument above. First, we have the estimate (13). For a zero $p_{0}$ of $\phi$ and a $\tilde{\phi}=\phi \circ h \in\left\{\phi \circ h_{i} \mid h_{i} \in \operatorname{Hol}(\tilde{X}, X)\right\}$, there are at most $2(\tilde{g}-1)$ possible points that a point $\tilde{p}_{0}$ with $h\left(\tilde{p}_{0}\right)=p_{0}$ can be. After determining $\tilde{\phi}$ and $\tilde{p}_{0}$, we have at most $2 g-1$ possible nonconstant holomorphic maps. Now, we obtain

$$
\sharp H o l(\tilde{X}, X) \leq(4(\tilde{g}-1) /(g-1)+1)^{2 \tilde{g}} \times 2(\tilde{g}-1) \times(2 g-1) .
$$




\section{REFERENCES}

[F-K] Farkas, H.M., Kra, I., Riemann surfaces, Springer-Verlag, New York, Heidelberg, and Berlin, 1980. MR 82c:30067

[F] de Franchis, M., Un teorema sulle involuzioni irrazionali, Rend. Circ. Mat. Palermo 36 (1913), 368.

[H-S] Howard, A., Sommese, A. J., On the theorem of de Franchis, Ann. Scoula. Norm. Sup. Pisa Cl. Sci. (4) 10 (1983), 429-436. MR 85k:32048

[H] Hurwitz, A., Über algebraischen Gebilde mit eindeutigen Transformation in sich, Math. Ann. 41 (1893), 403-442.

[I1] Imayoshi, Y., An analytic proof of Severi's theorem, Complex Variables Theory Appl. 2 (1983), 151-155. MR 85j:32037

[I2] Generalizations of de Franchis theorem, Duke Math. J. 50 (1983), 393-408. MR 85d:32051

[K] Kani, E., Bounds on the number of non-rational subfields of a function field, Invent. Math. 85 (1986), 185-198. MR 87i: 14021

[Mc] Macbeath, A. M., On a theorem of Hurwitz, Proc. Glasgow Math. Assoc. 5 (1961), 90-96.

[Mr1] Martens, H., A new proof of Torelli's theorem, Ann. of Math. (2) 78 (1963), 107-111. MR 27:2506

[Mr2] Observations on morphisms of closed Riemann surfaces, Bull. London Math. Soc. 10 (1978), 209-212. MR 58:1132

[Mr3] — Mappings of closed Riemann surfaces, Proc. Sympos. Pure Math. 49, Part 1 (1989), 531-539. MR 90i:14030

[T] Tanabe, M., On rigidity of holomorphic maps of Riemann surfaces, Osaka J. Math. 33 (1996), 485-496. MR 97i:32024

[W] Weyl, H., On generalized Riemann matrices, Ann. of Math. (2) 35 (1934), 714-729.

Department of Mathematics, Tokyo Institute of Technology, Ohokayama, Meguro, TOKYO, 152-8551, JAPAN

E-mail address: tanabe@math.titech.ac.jp 